\title{
A CORRECTION TO MY BOOK ON TOPOLOGICAL GROUPS
}

\section{ANDRÉ WEIL ${ }^{1}$}

On p. 28 of my monograph on topological groups, ${ }^{2}$ implicit use is made of the following proposition: "If $G$ is a topological group, and $g$ a closed invariant subgroup of $G$, then the factor-group $G / g$ cannot have a dimension greater than that of $G$." This is a very plausible but hitherto unproved result; the assertions on the dimension of a projective limit (IGTA, p. 28) must therefore partake of its conjectural nature. However, the results on the dimension of compact groups and of locally compact abelian groups (IGTA, chap. 5, §25, and chap. $6, \S 29)$ can be justified by dealing with a special case of that proposition; to do so is the purpose of the present note. Since no satisfactory theory of dimension exists except for "separable metrizable" spaces, ${ }^{3}$ it will be enough to consider the case of groups which are metrizable and separable. A compact group which satisfies these conditions will be called enumerably compact in this note; such a group has at most enumerably many irreducible linear representations; it follows from this, and from the results of IGTA, \$25, that it is the projective limit of a sequence of compact Lie groups. The result to be proved is now as follows.

THEOREM. Let $G$ be an enumerably compact group. Let $g_{0}$ be a closed invariant subgroup of $G$ such that $G / g_{0}$ is a Lie group. Let $U_{0}$ be a neighborhood of the unit-element in $G / g_{0}$, homeomorphic to the interior of a sphere; and let $X$ be the set of all elements of $G$, the image of which in $G / g_{0}$ is in $U_{0}$. Then $X$ is homeomorphic to the (topological) direct product $U_{0} \times g_{0}$.

Let $\phi$ be the canonical mapping of $G$ on the factor-group $G_{0}=G / g_{0}$, i.e., the mapping in which, to every element of $G$, corresponds its co-set with respect to $g_{0}$. As is well known, our theorem will be proved if we show that we can find in $G$ a system of representatives $U$ for $U_{0}$, i.e., a homeomorphic image $U=\psi\left(U_{0}\right)$ of $U_{0}$ in $G$ such that the inverse $\psi^{-1}$ of $\psi$ coincides on $U$ with $\phi$. In order to do that, we write $G$

Received by the editors August 7, 1944.

${ }^{1}$ Guggenheim Fellow.

${ }^{2}$ L'intégration dans les groupes topologiques et ses applications, Actualités Scientifiques et Industrielles, Hermann, Paris, 1940; this book will be quoted as IGTA. I am much indebted to H. Samelson for having first pointed out to me the conjectural nature of the result in question, and also for the following correction to the same book: on p. 26, lines 8 and 15, the word "invariant" should be omitted.

${ }^{3}$ Cf. W. Hurewicz and H. Wallman, Dimension theory, Princeton, 1941 (see the Appendix). 
as projective limit of a sequence of Lie groups $G_{n}=G / g_{n}$, where the $g_{n}$, for $n=0,1,2, \cdots$, are a sequence of closed invariant subgroups of $G$, beginning with the given subgroup $g_{0}$, such that $g_{n} \supset g_{n+1}$ for every $n$, and with no common element other than the unit-element of $G$. If we put $\gamma_{n}=g_{n+1} / g_{n}$, we can consider $\gamma_{n}$ as a subgroup of $G_{n}=G / g_{n}$; it is a closed invariant subgroup of $G_{n}$, and we have (by IGTA, §3) $G_{n}=G_{n+1} / \gamma_{n+1}$; let $\phi_{n}$ be the canonical mapping of $G_{n+1}$ on the factorgroup $G_{n}=G_{n+1} / \gamma_{n+1}$. We now define by induction, for every $n$, a homeomorphic image $U_{n}$ of $U_{0}$ in $G_{n}$ as follows. Assuming that $U_{n}$ has been defined, we may consider its inverse image by $\phi_{n}$, in $G_{n+1}$, as a fibre-space with the base-space $U_{n}$ and with fibres which are homeomorphic to $\gamma_{n+1}$; as $U_{n}$ is homeomorphic to $U_{0}$, that is, to the interior of a sphere of a certain dimension, it follows from this, by Feldbau's theorem, ${ }^{4}$ that this inverse image is homeomorphic to the topological product of $U_{n}$ and of $\gamma_{n+1}$, and that we can find in it a continuous system of representatives, which we call $U_{n+1}$, for the base $U_{n}$. Then $U_{n+1}$ is a homeomorphic image $U_{n+1}=\psi_{n}\left(U_{n}\right)$ of $U_{n}$ in $G_{n+1}$, and the inverse $\psi_{n}^{-1}$ of the homeomorphic mapping $\psi_{n}$ of $U_{n}$ on $U_{n+1}$ is no other than $\phi_{n}$. That being so, let $x_{0}$ be any point in $U_{0}$, and put $x_{n+1}=\psi_{n}\left(x_{n}\right)$ for $n=0,1,2, \ldots$; as we have $x_{n}=\phi_{n}\left(x_{n+1}\right)$ for every $n$, the sequence $\left(x_{0}, x_{1}, x_{2}, \cdots\right)$ defines a point $x$ in the projective limit $G$ of the sequence $G_{0}, G_{1}, G_{2}, \cdots$; if now we write $x=\psi\left(x_{0}\right), \psi$ is a mapping of $U_{0}$ in $G$, with the properties which were required for the proof of our theorem.

COROLLARY. If a factor-group of an enumerably compact group $G$ is a Lie group of dimension $n$, then $G$ itself has at least the dimension $n$.

The above proof suggests that the conjectural proposition mentioned at the beginning of this note is related to the following problem. Let $G$ be a topological group, $g$ a closed subgroup of $G$, and $H=G / g$ the homogeneous space determined by $G$ and $g$ (IGTA, §2), that is, the space of cosets of $g$ in $G$; can $G$ be considered as a fibrespace, with base $H$ and with fibres homeomorphic to $g$, according to any one of the known definitions of fibre-spaces? ${ }^{5}$ One may expect the answer to be in the affirmative, provided a point in $H$ has a system of neighborhoods which are all homotopic to zero (that is, contractible to a point) in $H$.

UNIVERSITY OF EREWHON, EREWHON, UT.

${ }^{4} \mathrm{~J}$. Feldbau, Sur la classification des espaces fibrés, C. R. Acad. Sci. Paris vol. 208 (1939) p. 1621.

${ }^{5}$ Cf., for example, R. H. Fox, On fibre-spaces, Bull. Amer. Math. Soc. vol. 49 (1943) p. 555. 